Chapter 14. Analysis of microplastics in food samples

\title{
Analysis of microplastics in food samples
}

\author{
Juan A. Conesa ${ }^{1}$ and Maria E. Iñiguez
}

Department of Chemical Engineering. University of Alicante. P.O. Box 99, 03080 Alicante (Spain). Phone: +(34) 965903867 Fax: +(34) 965903826.

\begin{abstract}
This chapter presents a compilation of the analytical techniques used to detect and analyse microplastics in food. A detailed description of microplastics found in different samples is provided as well as an estimate of the annual intake of these particles. A total of 22-37 milligrams of microplastics per year was found. The factors that can influence the presence of particles in food, especially table salt, are discussed, showing that a background presence of microplastics in the environment can explain a large amount of experimental data.
\end{abstract}

Keywords: microplastics, food, seafood, salt, beer, water, milk, juice

\section{Introduction}

Currently, a series of emerging pollutants are being studied by a number of scientists around the world due to the potential danger they present to the environment and human health. As time passes, these chemical or physical elements are increasingly affecting life and the environment. It is thus necessary to establish methodologies to analyse their presence, especially those that are not currently visible in certain environments.

Plastics are known to be durable, strong, lightweight and inexpensive, properties that

\footnotetext{
${ }^{1}$ Corresponding author email: ja.conesa@ua.es
} 
Chapter 14. Analysis of microplastics in food samples

make them suitable for a broad range of applications (Laist, 1987). Conversely, these same properties are what cause plastics to disperse easily, presenting environmental hazards, and turning plastic debris into a major worldwide pollution problem (Cózar et al., 2014; Iñiguez et al., 2016; Jambeck et al., 2015).

Global annual production of plastics amounts to around 360 million tonnes (PlasticsEurope and EPRO, 2016); in Europe alone, plastics production almost reached 62 million tonnes. It is important to add synthetic fibres to this figure: synthetic fibres are used in clothing, ropes, or other products, and together accounted for 61 million tonnes that same year. It is estimated that by 2020, plastics and synthetic fibres will together exceed 500 million tonnes per year, accounting for a $900 \%$ increase compared to production levels in 1980 .

Plastic items are used on a daily basis. Many computer and mobile phone components are made with polymers; bicycles, cars and motorbikes also have parts made of plastics; food wrapping is usually made of plastics. It has been estimated that $50 \%$ of plastics are used for single-use disposable applications, with the packaging and disposable consumer items (plates, glasses, bottles, shopping bags, among others) as the more representative.

The main reason plastics are hazardous for the environment is that they are highly resistant to degradation, so they can persist for long periods of time, probably for hundreds or thousands of years. Even so, plastics are exposed to various factors that fragment them into smaller pieces, turning them into microparticles below 5 millimetres in diameter that are widespread throughout the world.

It is important to understand that once used, most plastics end up in sinkholes such as rivers and seas until they empty into the oceans. For example, the friction from 
Chapter 14. Analysis of microplastics in food samples

washing machines causes polymer fibres in the clothes to be released, reaching sewers and eventually rivers. In general, water treatment plants cannot separate plastic microparticles, so they are passed on to consumers. Because water is used in most food processing, it is easy to assume that small plastic particles are consumed along with water, without there being any visible evidence of this consumption.

Studies on MPs have so far covered all major oceans. Peng et al. (Peng et al., 2020) studied the distribution of MPs in the oceans by analysing seawater, sediments, and animal samples. Results show that MPs is omnipresent in these oceans with few exceptions.

The impact of human exposure to microplastics has not yet been understood, leading to many unresolved questions (Sarria-Villa and Gallo-Corredor, 2016). For this reason, studies are now emerging on the presence and influence of microplastics, especially in the marine food chain because if they reach the sea, they are consumed by unicellular organisms such as zooplankton, thus entering the food chain until they are consumed by humans. Table salt has also been given importance (Chang et al., 2020) and studies have lately focused on rivers; as far as food is concerned, studies have been conducted on water (Kosuth et al., 2018), honey (Liebezeit and Liebezeit, 2014, 2013; Mühlschlegel et al., 2017), beer (Kosuth et al., 2018), fish (Li et al., 2015), though no efforts have been made concerning other processed products such as soft drinks, which are consumed en masse by human beings.

The presence of micro- (MPs) and nanoplastics in animals and food products that are part of the human food chain is a matter of concern, due to their possible effects on the human body. Today, MPs are ubiquitous in the environment and mainly come from single-use plastics, fishing gear, clothing and cosmetics, paints, tyres and urban 
Chapter 14. Analysis of microplastics in food samples

dust (Browne et al., 2011). Due to their generally low density, it is assumed that a significant portion of plastics discharged into rivers can reach the sea.

Several studies tried to determine the toxicity of MPs performing in vivo studies using different mammalian organisms (Rubio et al., 2019). In these studies, the permeability of the epithelial membrane to MPs was evaluated. These authors observed low percentages of absorption of polymeric particles up to $150 \mu \mathrm{m}$, confirming that the intestinal epithelium is an important and robust barrier against these types of materials.

Marine products are commonly consumed by people (e.g. fish, bivalves, salt), and they are definitely contaminated by MPs. As pointed out in previous studies (Bouwmeester et al., 2015; Yang et al., 2015) it is important to investigate the risk of MPs being transferred from sea products to humans through the food chain, evaluating potential health risks for humans.

The most important studies on the presence of MPs in food are related to edible animal species (mainly sea fish, but also molluscs, crustaceans, birds, turtles and chicken), beer and water, and also to some food products (canned fish, table salt, sugar and honey). Different studies have been carried out, some of which conclude that there is no significant contamination, and others do not find safety hazards (Toussaint et al., 2019).

With respect to marine species, MPs can be directly ingested by animals, or trophic transfer may occur (Carbery et al., 2018; Toussaint et al., 2019). The latter can lead to biological accumulation, with higher concentrations in upper trophic levels than lower ones (Carbery et al., 2018). In this way, MPs have been detected in the gastrointestinal tract of fish, bivalves, crustaceans and turtles (Toussaint et al., 2019). Almost all studies report that the most abundant form of MPs is fibres (called microfibres) versus microballs. 
Chapter 14. Analysis of microplastics in food samples

Different studies mention that treated wastewater is one of the most important sources of MPs to the oceans. Recently, a study has been published (Hidayaturrahman and Lee, 2019) on the efficacy of traditional methods of sewage treatment in terms of the presence of microplastics. This study investigated the elimination of microplastics from different treatment stages in three WWTP (Wastewater Treatment Plant) and examined the efficacy of tertiary treatment that was performed by coagulation and different technologies such as ozonation, use of membrane disk filters and rapid filtration of sand. The results showed that primary and secondary treatment processes effectively eliminate microplastics from wastewater with efficiency ranging from $75 \%$ to $91.9 \%$. Elimination efficiency increased further to $>98 \%$ after tertiary treatment. Microbeads and fragments were the main types of microplastics found in all wastewater samples. Even so, microplastics were still found in a high concentration in the final effluent, especially in the plant that used membrane filters as a tertiary treatment.

\section{Analytical techniques used to isolate, count, and identify MPs}

There is abundant literature on the presence of MPs in food, based on a wide range of methods to isolate, count and analyses of MPs in the different foods. For solid nonsoluble species (fish, meats, etc.), the procedure usually begins with the dissection of the animal under a stereoscopic microscope. The MPs are then visually identified and transferred to a covered Petri dish, where the particles are then counted. The polymer is eventually identified using a micro-FTIR, or sometimes Raman spectroscopy on selected fibres.

\subsection{Pre-treatment and separation of MPs in food}

Many different techniques have been used for the pre-treatment of some food samples to separate the microplastics. Some of the most relevant are: 
Chapter 14. Analysis of microplastics in food samples

- Honey and sugar: cellulose fibres and chitin fragments are decolorized by applying $\mathrm{H}_{2} \mathrm{O}_{2}$ pre-treatment. The fibres appear to be transparent or can be stained by using fuchsine and rose Bengal. The uncoloured fibres are considered to be synthetic polymers (Liebezeit and Liebezeit, 2013).

- Salt: dissolution in ultrapure water and filtration. Filters should have a small pore size to prevent underestimations (Iñiguez et al., 2017). The particles are then identified using an optical microscope.

- Beer: Fibres, fragments and granules are isolated simply by filtering. Rose Bengal can be used to distinguish between synthetic and natural polymers.

- Bottled and tap water: reported detection method based on staining using Nile red (for particles $>6.5 \mu \mathrm{m}$ ) and further identification by FTIR (for particles $>100 \mu \mathrm{m})$. Nile red is adsorbed on plastic and is detected by fluorescence.

\subsection{Sample preparation methods}

- Filtration. This is the simplest method for isolating MPs. It is a low-cost technique that can be useful to categorise particles by size. It is only suitable for water and non-viscous beverages, although its application can be extended to soluble species such as table salt and sugar. Its main limitation is that the filter can be easily clogged if the pore size is small, and special care should be taken to retain all target particles using appropriate equipment.

- Flotation and sedimentation. Also represent simple and low-cost techniques to isolate the fibres, and to separate plastics from inorganic matter. Their main drawback is that the plastic particles should be previously detached from matrix materials. 
Chapter 14. Analysis of microplastics in food samples

- Matrix digestion by acidic or alkaline chemical digestion. This technique is appropriate for animal tissues and organs, but it needs to be optimised to digest all organic matter and, in some cases, it can damage or destroy the plastic particles.

- Matrix digestion by enzymatic treatment. This digestion leads to a generally lower degree of damage to polymers compared to alkaline or acidic treatment. It is appropriate for many biological matrices but is rather expensive and unpredictable to the extent that the enzymatic activity highly depends on the matrix.

- Oil extraction. This technique is valid for separating plastics from inorganic matter in aqueous media. The analytes need to be sufficiently hydrophobic to be extracted by the oil, and damage can occur if the temperature is excessively high.

\subsection{Methods used to identify MPs}

- Micro-FTIR spectroscopy. The most widespread method in food sample analysis. The IR spectra of unknown microplastics sample can be compared with the IR spectra of known polymers available from IR spectra database. It is a selective identification technique with reproducible results. Requires sample preparation (clean-up), as the MPs have to be taken one by one, but it is not destructive.

- Micro-Raman spectroscopy. This technique is equally widespread. It also requires sample preparation, and it identifies isolated particles. The main drawbacks are the samples' autofluorescence that may mask the signal. Also the presence of colour in the samples can interfere with the identification of plastic 
Chapter 14. Analysis of microplastics in food samples

type. The technique may not be reliable on weathered samples, degraded samples or mixed polymer samples. Raman spectroscopy has been used for the identification of MPs from wastewater (Fortin et al., 2019), and also surface water and sediments (Kazour et al., 2019).

- $\quad$ Thermal analysis, combining pyrolysis and gas chromatography with the MS detector (Py-GC-MS). The technique is based on the analysis of the microplastics thermal degradation products and is able to identify both the polymer type and some organic additives used in the formulation. Usually this is done in one single run and without the use of solvents thus avoiding background contamination. The Py-GC-MS does not allow determining the number, size or shape of the particles. The detection limit corresponds to the range of particles measuring several millimetres, which can be a major problem. This is a destructive technique does not provide data on the size and shape of the plastic fragments examined and only give mass concentration results.

- Fluorescent tagging with Nile red. This technique improves the counting of MPs, as it makes most synthetic polymers. Dye is added to the samples in a very low concentration $(10 \mathrm{mg} / \mathrm{L})$ and is able to coloring different types of polymers and synthetic textiles. In the literature, excitation wavelengths used for Nile Red stained microplastics vary from 365 to $510 \mathrm{~nm}$. The particle size should be between $20 \mu \mathrm{m}$ and $1 \mathrm{~mm}$. The residual organic matter in the matrix may get stained and produce false positives.

- Time-of-flight secondary ion mass spectrometry (ToF-SIMS). This is a high spatial resolution imaging technique suitable for mixture of particles, but the technique is complex to use and very expensive. Sometimes the identification complicated by weathering or by surface contaminants. The sample must also be 
Chapter 14. Analysis of microplastics in food samples

vacuum-compatible. This technique has been used to analyse polyethylene microplastics in seawater (Jungnickel et al., 2016). Mass spectra has been published for the main polymers found in MPs (Kern et al., 2019).

- Hyperspectral imaging technology. This technique has been used recently to detect microplastics in seawater rapidly(Shan et al., 2019). It is able to identify with a single image polymers greater than $0.2 \mathrm{~mm}$.

\subsection{Possible contamination from food packaging plastics:}

Adding to the MPs contained in the food items themselves, another possible source of micro- and nanoplastic particles is food packaging. On the one hand, some of the chemicals used in plastics manufacture (such as residual monomers, additives and pigments) might migrate from a plastic package to the food product (Toussaint et al., 2019). Conversely, it is unlikely a spontaneous release of micro- and nanoplastic particles from the packaging material. Plastic food packaging could break into small pieces and contaminate the food products if wrongly handled. But, in these cases, it is expected that plastic fragments would be of a relatively big size and could easily be identified visually, which would ensure their removal before consumption.

Iñiguez et al (Iñiguez et al., 2017) studied the effect of packaging on MPs levels in different salts. Their results showed similar microplastic content before (185 particles/kg) and after (120 particles/kg) they were packaged. Their results also showed that grinding did not affect the presence of MPs in salt samples, as they found that samples before and after being grounded presented similar MP contents (115 vs. 120 particles/kg). 
Chapter 14. Analysis of microplastics in food samples

\section{Risk assessment}

The impact on human health is usually related to the fact that they may carry potentially toxic chemicals and microorganisms (Mato et al., 2001; Oberbeckmann et al., 2015; Rios et al., 2007). MPs have the ability to adsorb (or absorb) these hazardous chemical pollutants, such as metals and toxins, from the environment and transfer them to food products, that are finally consumed by humans (Brennecke et al., 2016; Iñiguez et al., 2017; Koelmans et al., 2014; Talvitie et al., 2015; Yang et al., 2015).

Vethaak and Leslie (Dick Vethaak and Leslie, 2016) mention that plastic debris can be regarded as complex cocktails of contaminants, including chemical additives, residual monomers and ambient chemical substances adsorbed to plastic surface. Many of these pollutants (such as bisphenol A, phthalates and some brominated flame retardants) are considered as endocrine disruptors that can cause severe health problems.

In this way, the risk evaluation of MPs in food products should focus not only on the effect of MPs themselves, but especially on the effects of the chemical pollutants adsorbed by MPs, that may possibly be released with a negative impact on animals and the environment. In this line, Gassel et al. (Gassel et al., 2013) suggested that the most likely source of elevated concentrations of the nonylphenol - a surfactant commonly found in detergents and that acts as an endocrine disruptor - plastic additive in fish tissues was ingestion of microplastics.

Nevertheless, these studies are at a preliminary stage and we dispose of very few studies on MP levels in foodstuff and their effects on human health (Rainieri and Barranco, 2019). The effect of microplastics on living organisms is a highly controversial matter. Some studies report that microplastics produce adverse effects for aquatic organisms ( $\mathrm{Lu}$ et al., 2016; Rochman et al., 2013), while others report the opposite (Bakir et al., 2016). 
Chapter 14. Analysis of microplastics in food samples

Generally, the effects are largely related to the size of MPs. In this way, particles over $150 \mu \mathrm{m}$ are probably not absorbed by organisms, and would simply produce limited inflammatory effects; by contrast, smaller particles might cause the whole organism to be exposed, and fractions as small as $1.5 \mu \mathrm{m}$ might penetrate the organs. Nanoplastics are thus the greatest cause for concern (Bouwmeester et al., 2015).

On the other hand, the presence of MPs in the gastrointestinal tract of different fish species does not imply human exposure, as this organ is usually not consumed. Nevertheless, other seafood species that are consumed whole (e.g. some molluscs and crustaceans, and small or juvenile phases of fish) pose a biggest warning to seafood contamination. MPs have been detected in the stomachs of many different fish around the world, and in the gastrointestinal tract and liver of anchovies and sardines that are sometimes consumed whole (Barboza et al., 2018).

\section{MPs in different food and estimation of total ingestion}

\subsection{MPs in marine and terrestrial species}

The number of published studies on contamination of microplastics in different animal species, edible and inedible, has not stopped growing since 2010. Most studies have focused on the analysis of fish and marine mammals (dolphins, sea lions or seals), although some bivalves have also been investigated, such as mussels, oysters and scallops. The works also include some seabirds (cormorants, seagulls or puffins), and other minor species.

A study by Boerger et al. (Boerger et al., 2010), based on the analysis of the stomach content of 670 fish belonging to five mesopelagic species (living between 200$1000 \mathrm{~m}$ deep) and one epipelagic (living between the surface and $200 \mathrm{~m}$ ), confirmed the intake of plastics by the fish and estimated the amount of plastic ingested in 1375 pieces. 
Chapter 14. Analysis of microplastics in food samples

Lönnstedt and Eklöv (Lönnstedt and Eklöv, 2016) experimentally demonstrated that the intake of plastic particles of less than $5 \mathrm{~mm}$ by fish larvae can cause reproductive problems and threats to their survival.

Tanaka et al. (Tanaka et al., 2013) also demonstrated the impact of these pollutants in marine birds as it analysed the stomach content of different species and found plastic particles in all of them.

The main result of the publications consulted is that microplastic contamination in the studied species is a fact, since its presence has been described in more than 690 species, mainly marine, 200 of which are edible (Carbery et al., 2018).

Similarly, filter organisms such as bivalves are affected. The study of Mathalon and Hill (Mathalon and Hill, 2014) first presented data on the presence of MPs in bivalves, with significantly more microplastics enumerated in farmed mussels compared to wild mussels (180 vs. 100 particles/mussel, approximately).

In a study conducted by $\mathrm{Li}$ et al. ( $\mathrm{Li}$ et al., 2015), the abundance and types of microplastics present in nine of the most commercialized bivalve species in Shanghai, China, was assessed. From the results, they concluded that there were high levels of contaminants in bivalves.

An interesting study (Hall et al., 2015) has also shown that corals are indirectly or directly fed microplastics, although they have not been able to demonstrate how these microplastics affect coral growth.

Lwanga et al. (Huerta Lwanga et al., 2017) assessed micro- and macro-plastics contamination in soil, earthworm casts, chicken faeces, crops and gizzards (used for human consumption), showing that the number of MPs per gram was increasing in the order: soil $(0.87 \pm 1.9$ particles $/ g)<$ earthworm cast $(14.8 \pm 28.8$ particles $/$ ga $)<$ chicken 
Chapter 14. Analysis of microplastics in food samples

faeces $(129.8 \pm 82.3$ particles/g), finding no significant correlations between any of them.

No studies have been found in literature concerned with analyzing MPs in other types of meat, such as pork, goat, sheep, and veal.

\subsection{MPs in honey}

MPs content in honey was first investigated by Liebezeit (Liebezeit and Liebezeit, 2013), which found 40 to 660 fibres/kg and 0 to 38 fragments/kg in German honeys. The authors also found black carbon particles originating from the smoking of the hives, a common practice in bee-keeping to calm bees before harvesting.

On the other hand, Mühlschlegel et al. (Mühlschlegel et al., 2017) investigated the contamination of honey from Switzerland by MPs. The authors concluded that the composition and quantity of particles and fibers detected in honey samples did not pose a health risk.

\subsection{MPs in liquids and soluble food samples}

To prepare this chapter, different foods were subjected to an analysis of microplastics content. A similar method to that presented above was followed, adapting the methodology to each specific sample. The process basically consisted in dissolving the solid samples, centrifugation and filtration through a $5 \mu \mathrm{m}$ pore size vacuum system (Iñiguez et al., 2017). Different foods were analysed, together with special salt samples discussed later. The results are summarised in the table below. Figure 1 shows some images of the isolated fibres retrieved from some of the samples analysed in this work.

As we can see, MPs were ubiquitous and present in all tested products. The levels of MPs found in sugar were comparable to the levels of MPs found in salt 
Chapter 14. Analysis of microplastics in food samples

(Iñiguez et al., 2017). However, the average consumption of sugar is unfortunately much higher than that of salt (120 vs. $5 \mathrm{~g}$ /day) meaning that annual exposure is much greater. Apple juice, wine, beer and other beverages presented similar levels, within the range of 15- 45 particles/L, and milk products presented a slightly lower presence (4-21 particles/L). MPs content in beer has been reported previously (Liebezeit and Liebezeit, 2014), showing levels of 16-254 particles/L (sum of fibres, fragments and granules). In addition, Kosuth et al. (Kosuth et al., 2018) tried to correlate the presence of MPs in beer with the levels of MPs found in the water used for manufacturing the product. They concluded that, while both the municipal tap water and the beers analysed all contained anthropogenic particles, there is no correlation between the two, indicating that any contamination within the beer is not just from the water used to brew the beer itself.

Table 1. Concentration of microplastics (number of particles) found in different soluble and liquid food samples.

\begin{tabular}{|c|c|}
\hline Sample & $\begin{array}{c}\text { Unit concentration } \\
\text { (MPs/L) or (MPs/kg) }\end{array}$ \\
\hline Apple juice & 21 \\
\hline White wine & 36 \\
\hline Red wine & 15 \\
\hline Beer & 45 \\
\hline Sugar & 230 \\
\hline Whole fat milk & 21 \\
\hline Semi-skimmed milk & 15 \\
\hline Skimmed milk & 4 \\
\hline Soda & 4 \\
\hline Vinegar & 15 \\
\hline Sunflower oil & 6 \\
\hline
\end{tabular}


Chapter 14. Analysis of microplastics in food samples

\begin{tabular}{|c|c|}
\hline Soda with red wine (sangria) & 5 \\
\hline Tonic water & 2 \\
\hline Salt vacuum (Pharma grade) & 4 \\
\hline Salt vacuum (in pellets) & 40 \\
\hline Dead sea salt & 225 \\
\hline
\end{tabular}

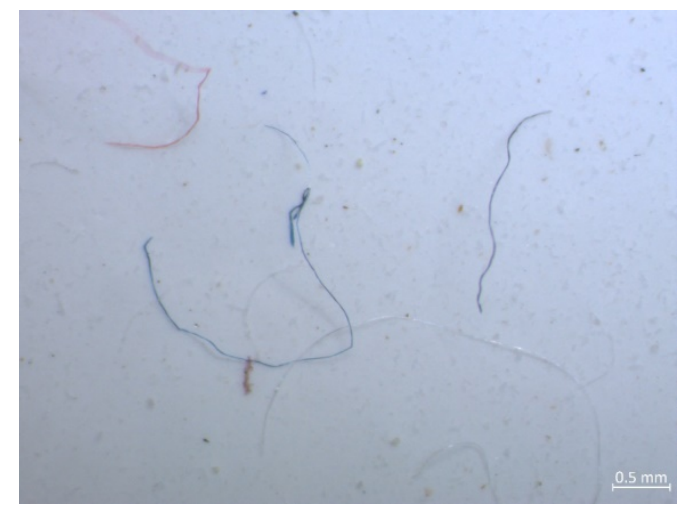

Sugar

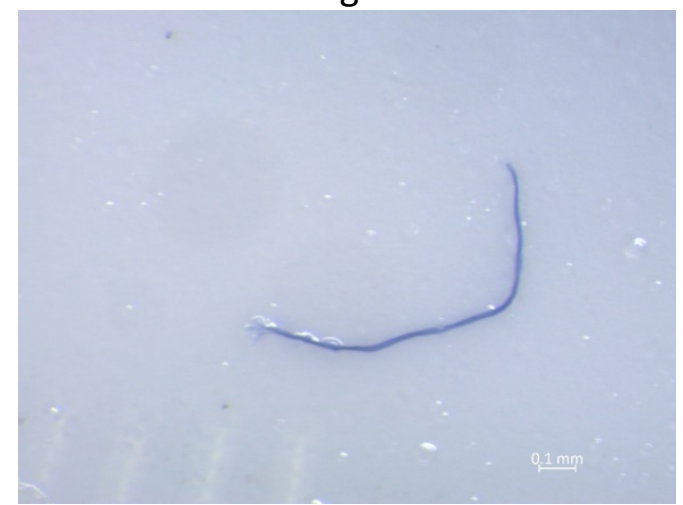

Milk (whole fat)

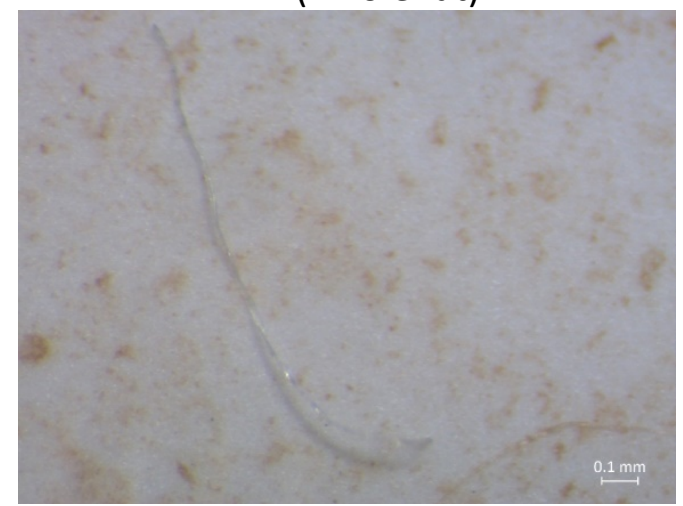

Apple juice

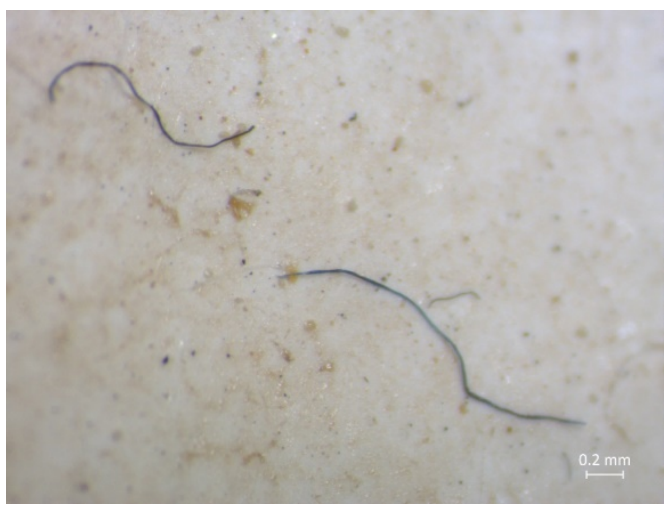

Beer

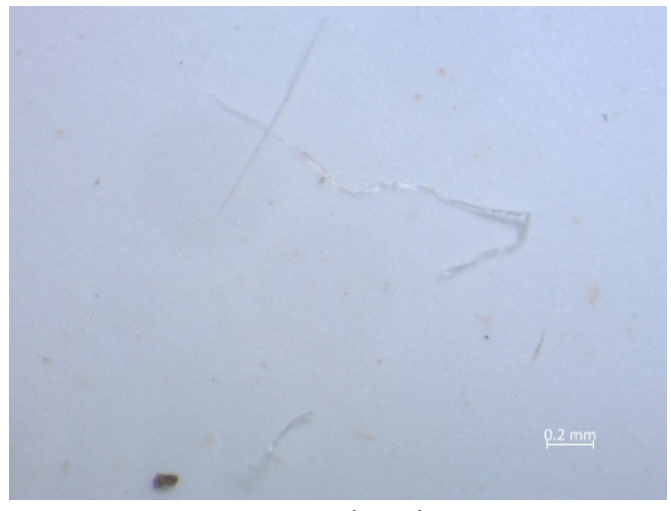

Wine (red)

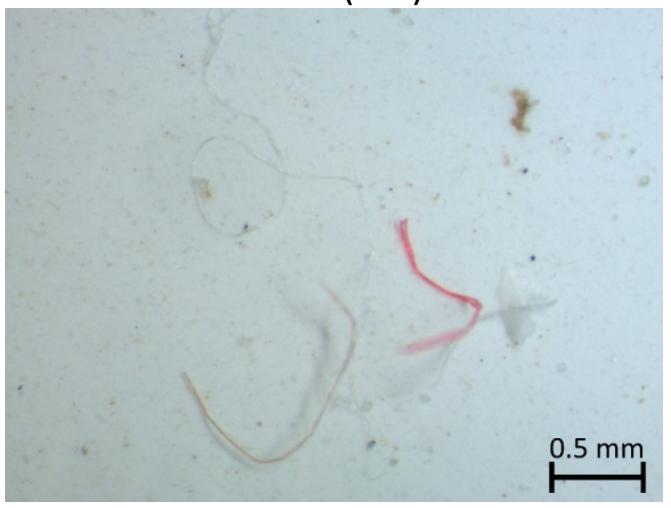

Dead sea salt 
Chapter 14. Analysis of microplastics in food samples

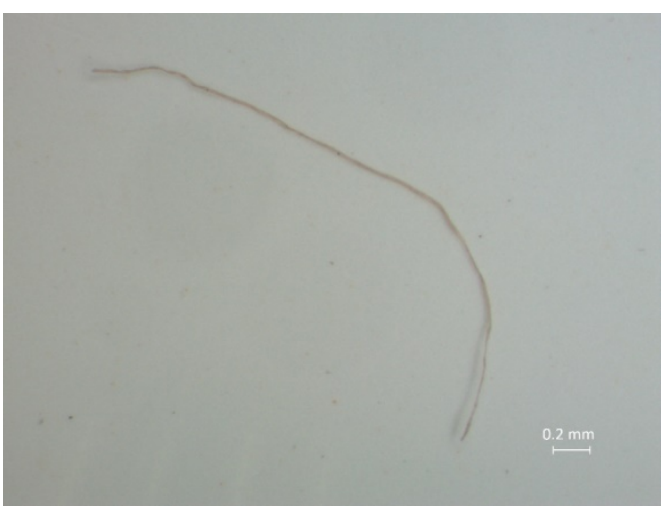

Salt vacuum (Pharma grade)

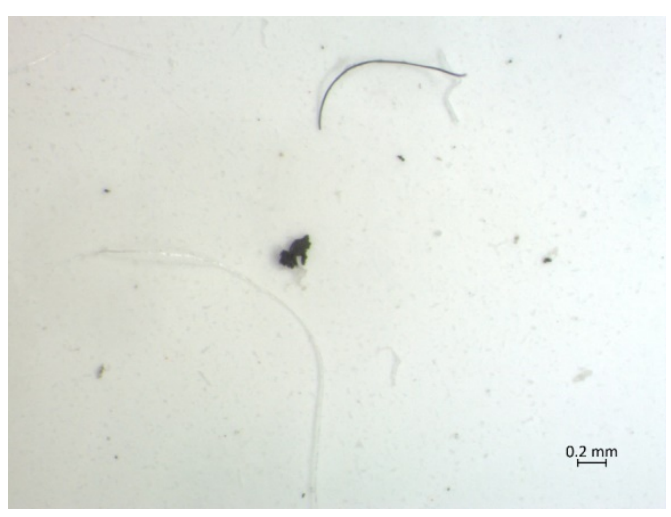

Salt vacuum

Figure 1. Micro-photographs of some microplastics found in the analysed samples.

Studies focusing on the determination of MPs in milk have not been found in the literature, which will probably contribute significantly to an increase in global exposure to these particles. From data in Table 1, an impact of 4-21 particles/L can be expected.

\subsection{MPs in table and special salts}

In previous work, Iñiguez et al. indicated that a possible reason for MPs presence in sea salt was the background presence of MPs in the environment, because the MPs content in Spanish sea salt (50-280 particles $/ \mathrm{kg}$ ) was not significantly different from that found in other types of salt (sea salts versus well salts). Figure 2 shows the elements/kg found in salts of different origin (maximum, minima and average value), where we can clearly observe similar levels of MPs. 
Chapter 14. Analysis of microplastics in food samples

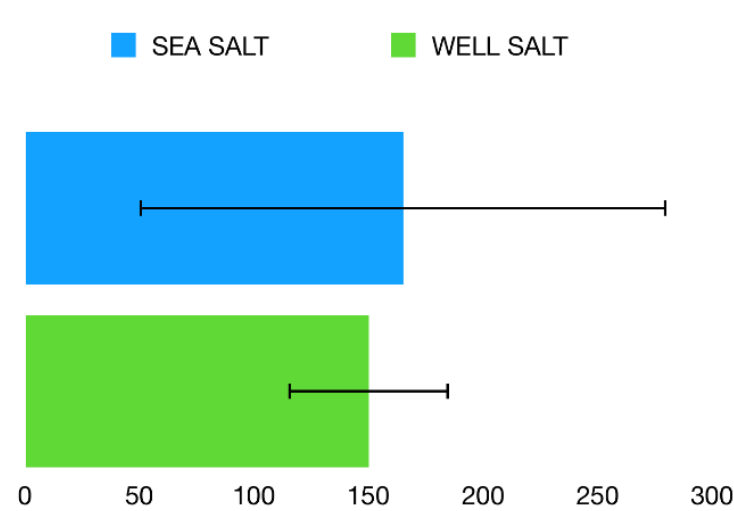

Figure 2. Number of microplastic particles found per $\mathrm{kg}$ of salt according to its origin (well or sea salts). Average values and measure intervals (Iñiguez et al., 2017).

Compared to this study, much lower amounts of MPs (a range of 0-10 particles $/ \mathrm{kg}$ ) were present in different salt samples purchased from a Malaysian market (Karami et al., 2017). In this latter study, the salts were produced in eight countries and the source of the salt- hosted MPs was not addressed. Nevertheless, a $150 \mu \mathrm{m}$ pore size filter was used so the amount of MP presence was probably underestimated. In a different study, Kosuth (Kosuth et al., 2018) found "anthropogenic debris" at concentrations of 47-806 particles/kg in table salts purchased in the USA, though no investigation into the possible causes of the salt pollution was conducted. Lastly, Renzi and Blasǩkovic (Renzi and Blašković, 2018) mentioned dense human activities around salt production sites and the polluted Mediterranean Sea to be the cause of concentrations of 1000-20000 particles/kg detected in Italian and Croatian fine sea iodate salts. Among the mentioned human activities is the fact that coastal area is highly overexploited by human settlements and also the fact that salt flats are usually not far from urban zones and from river inputs.

In a recent test, we studied the presence of MPs in different salt samples. For that, we obtained samples of salt pharmaceutical grade (used for preparing saline medium for hospital purposes), and other vacuum-type salts. The production of vacuum 
Chapter 14. Analysis of microplastics in food samples

salts differs from the traditional means of production. These salts are obtained from a chemically purified brine (salt water), via a process of controlled thermal compression and vacuum evaporation, and they are then solidified and crystallised into a spherical shape with a minimum content of $99.7 \%$ pure sodium chloride $(\mathrm{NaCl})$. The results of the presence of MPs are shown in Table 1. These results reveal that MP levels in these salts is very low, indicating that the previously found (Iñiguez et al., 2017) background level of MPs can be eliminated using appropriate techniques.

In this sense, we would like to conclude on the MP levels found in the different studies. Generally, high MP content values (we would say $>400$ particles $/ \mathrm{kg}$ ) were observed in salts from highly contaminated areas, which would indicate that a certain correlation between level of contamination and MP levels exists. On the other hand, a background level exists (around 200 particles $/ \mathrm{kg}$ ) that could not be eliminated unless sophisticated (and expensive) techniques were used.

\subsection{Estimation of total ingestion of MPs contained in food and drinks}

In a very recent study, Cox et al. (Cox et al., 2019) estimated the total ingestion of MPs, using 402 datasets from 26 studies. The authors reported an annual microplastics consumption range between 39,000 and 52,000 particles, depending on people's age and size. These estimates increased to 74,000 and 121,000 when air inhalation was taken into account. Surprisingly, Cox et al. mention that water intake by only bottled sources could account for an additional ingestion of 90,000 microplastics annually.

In their study, Cox et al. demonstrate that air, bottled water, and seafood consumption accounted for the large majority of microplastic intake. Also, the consumption of added sugars (including honey) contributed in a notable number of MPs to total consumption values. Figure 3 shows the maximum and minimum values used in the study for 
Chapter 14. Analysis of microplastics in food samples

different matrices.

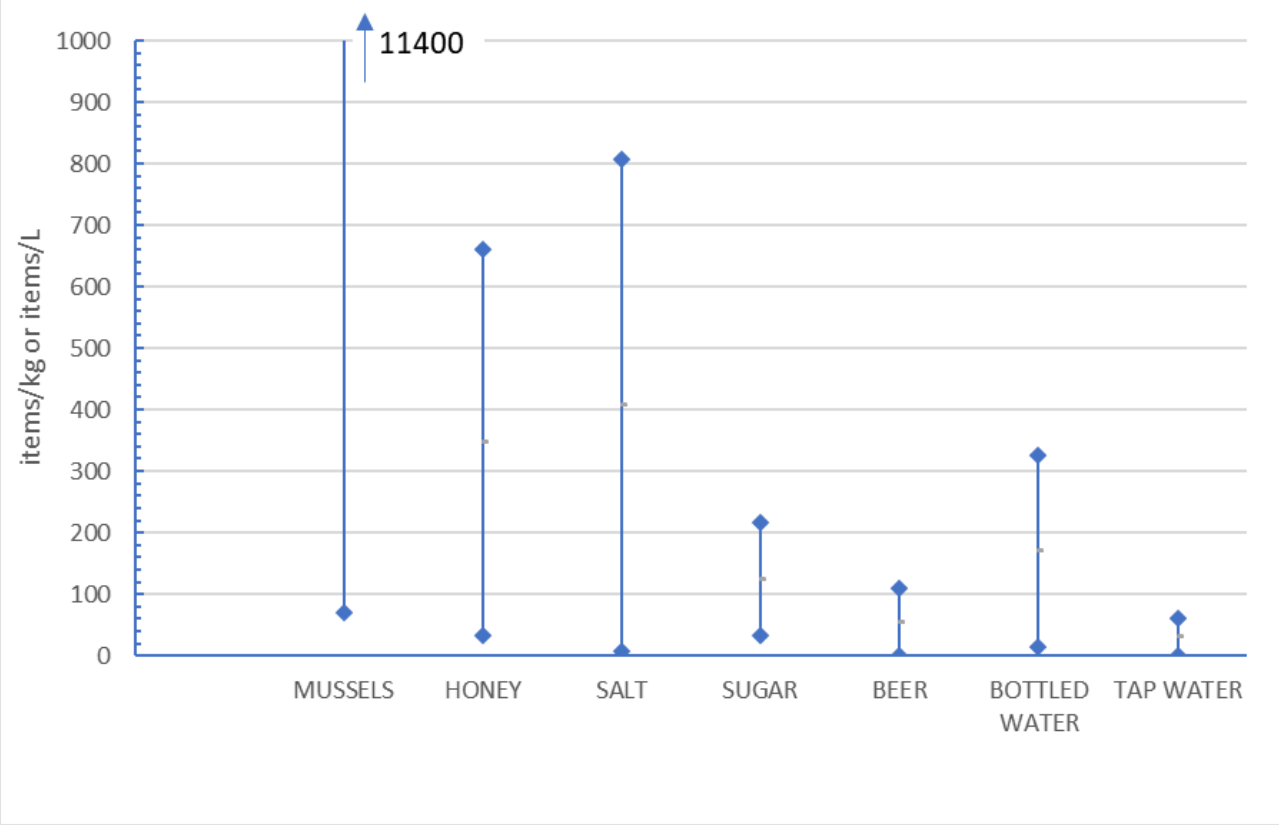

Figure 3. Values of $\mathrm{MPs} / \mathrm{kg}$ or $\mathrm{MPs} / \mathrm{L}$ used to estimate total ingestion (Cox et al., 2019).

Based on an average size of $3 \mathrm{~mm}$ (length) per 1-2 $\mathrm{mm}$ in diameter, and assuming these particles have a density of $1.3 \mathrm{~g} / \mathrm{L}$ (value corresponding to PET), a weight of 0.3 micrograms can be established per MP. This accounts for a total ingestion of 22-37 milligrams of microplastics per year, based on the total ingestion data commented previously. This data is very different from that reported by the University of Newcastle (Senathirajah and Palanisami, 2019), which mentions a weekly intake of more than 5 grams of plastics, although this data has not been published in scientific literature.

Concerning nanoplastics, few studies have evaluated their presence in the human food chain. This fact could be due either to a lack of interest, or to the absence of validated and standardised sampling and analytical methodologies. No peer reviewed study has yet unambiguously demonstrated the presence of nanoplastics in food products (Toussaint et al., 2019). 
Chapter 14. Analysis of microplastics in food samples

\section{Acknowledgements}

Support for this work was provided by the CTQ2016-76608-R project from the Ministry of Economy, Industry and Competitiveness (Spain) and by the University of Alicante under the project UAUSTI18-06.

\section{References}

Bakir, A., O’Connor, I.A., Rowland, S.J., Hendriks, A.J., Thompson, R.C., 2016.

Relative importance of microplastics as a pathway for the transfer of hydrophobic organic chemicals to marine life. Environ. Pollut. 219, 56-65. doi:10.1016/j.envpol.2016.09.046

Barboza, L.G.A., Dick Vethaak, A., Lavorante, B.R.B.O., Lundebye, A.-K.K., Guilhermino, L., 2018. Marine microplastic debris: An emerging issue for food security, food safety and human health. Mar. Pollut. Bull. 133, 336-348. doi:10.1016/j.marpolbul.2018.05.047

Boerger, C.M., Lattin, G.L., Moore, S.L., Moore, C.J., 2010. Plastic ingestion by planktivorous fishes in the North Pacific Central Gyre. Mar. Pollut. Bull. 60, 22752278. doi:10.1016/j.marpolbul.2010.08.007

Bouwmeester, H., Hollman, P.C.H., Peters, R.J.B., 2015. Potential Health Impact of Environmentally Released Micro- and Nanoplastics in the Human Food Production Chain: Experiences from Nanotoxicology. Environ. Sci. Technol. 49, 8932-8947. doi:10.1021/acs.est.5b01090

Brennecke, D., Duarte, B., Paiva, F., Caçador, I., Canning-Clode, J., 2016. Microplastics as vector for heavy metal contamination from the marine 
Chapter 14. Analysis of microplastics in food samples

environment. Estuar. Coast. Shelf Sci. 178, 189-195.

doi:10.1016/J.ECSS.2015.12.003

Browne, M.A., Crump, P., Niven, S.J., Teuten, E., Tonkin, A., Galloway, T., Thompson, R., 2011. Accumulation of microplastic on shorelines woldwide: Sources and sinks. Environ. Sci. Technol. 45, 9175-9179. doi:10.1021/es201811s

Carbery, M., O’Connor, W., Palanisami, T., 2018. Trophic transfer of microplastics and mixed contaminants in the marine food web and implications for human health. Environ. Int. 115, 400-409. doi:10.1016/j.envint.2018.03.007

Chang, X., Xue, Y., Li, J., Zou, L., Tang, M., 2020. Potential health impact of environmental micro- and nanoplastics pollution. J. Appl. Toxicol. 40, 4-15. doi:10.1002/jat.3915

Cox, K.D., Covernton, G.A., Davies, H.L., Dower, J.F., Juanes, F., Dudas, S.E., 2019. Human Consumption of Microplastics. Environ. Sci. Technol. acs.est.9b01517. doi:10.1021/acs.est.9b01517

Cózar, A., Echevarría, F., González-Gordillo, J.I., Irigoien, X., Úbeda, B., HernándezLeón, S., Palma, Á.T., Navarro, S., García-de-Lomas, J., Ruiz, A., 2014. Plastic debris in the open ocean. Proc. Natl. Acad. Sci. 111, 10239-10244.

Dick Vethaak, A., Leslie, H.A., 2016. Plastic Debris Is a Human Health Issue. doi:10.1021/acs.est.6b02569

Fortin, S., Song, B., Burbage, C., 2019. Quantifying and identifying microplastics in the effluent of advanced wastewater treatment systems using Raman microspectroscopy. Mar. Pollut. Bull. 149, 110579. 
Chapter 14. Analysis of microplastics in food samples

doi:10.1016/j.marpolbul.2019.110579

Gassel, M., Harwani, S., Park, J.-S., Jahn, A., 2013. Detection of nonylphenol and persistent organic pollutants in fish from the North Pacific Central Gyre. Mar. Pollut. Bull. 73, 231-242. doi:10.1016/J.MARPOLBUL.2013.05.014

Hall, N.M., Berry, K.L.E., Rintoul, L., Hoogenboom, M.O., 2015. Microplastic ingestion by scleractinian corals. Mar. Biol. 162, 725-732. doi:10.1007/s00227$015-2619-7$

Hidayaturrahman, H., Lee, T.G., 2019. A study on characteristics of microplastic in wastewater of South Korea: Identification, quantification, and fate of microplastics during treatment process. Mar. Pollut. Bull. 146, 696-702. doi:10.1016/j.marpolbul.2019.06.071

Huerta Lwanga, E., Mendoza Vega, J., Ku Quej, V., Chi, J. de los A., Sanchez del Cid, L., Chi, C., Escalona Segura, G., Gertsen, H., Salánki, T., van der Ploeg, M., Koelmans, A.A., Geissen, V., 2017. Field evidence for transfer of plastic debris along a terrestrial food chain. Sci. Rep. 7, 14071. doi:10.1038/s41598-017-14588-2

Iñiguez, M.E., Conesa, J.A., Fullana, A., 2017. Microplastics in Spanish Table Salt. Sci. Rep. 7, 1-7. doi:10.1038/s41598-017-09128-x

Iñiguez, M.E., Conesa, J.A., Fullana, A., 2016. Marine debris occurrence and treatment: A review. Renew. Sustain. Energy Rev. 64, 394-402.

Jambeck, J.R., Geyer, R., Wilcox, C., Siegler, T.R., Perryman, M., Andrady, A., Narayan, R., Law, K.L., 2015. Plastic waste inputs from land into the ocean. Science (80-. ). 347, 768-771. doi:10.1126/science.1260352 
Chapter 14. Analysis of microplastics in food samples

Jungnickel, H., Pund, R., Tentschert, J., Reichardt, P., Laux, P., Harbach, H., Luch, A., 2016. Time-of-flight secondary ion mass spectrometry (ToF-SIMS)-based analysis and imaging of polyethylene microplastics formation during sea surf simulation. Sci. Total Environ. 563-564, 261-266. doi:10.1016/j.scitotenv.2016.04.025

Karami, A., Golieskardi, A., Keong Choo, C., Larat, V., Galloway, T.S., Salamatinia, B., 2017. The presence of microplastics in commercial salts from different countries. Sci. Rep. 7, 46173. doi:10.1038/srep46173

https://www.nature.com/articles/srep46173\#supplementary-information

Kazour, M., Jemaa, S., Issa, C., Khalaf, G., Amara, R., 2019. Microplastics pollution along the Lebanese coast (Eastern Mediterranean Basin): Occurrence in surface water, sediments and biota samples. Sci. Total Environ. 696, 133933. doi:10.1016/j.scitotenv.2019.133933

Kern, S., Kern, C., Rohnke, M., 2019. Mass spectra database of polymers for bismuthcluster ToF-SIMS. Surf. Sci. Spectra 26, 025003. doi:10.1116/1.5096485

Koelmans, A.A., Gouin, T., Thompson, R., Wallace, N., Arthur, C., 2014. Plastics in the marine environment. Environ. Toxicol. Chem. 33, 5-10. doi:10.1002/etc.2426

Kosuth, M., Mason, S.A., Wattenberg, E. V., 2018. Anthropogenic contamination of tap water, beer, and sea salt. PLoS One 13, e0194970. doi:10.1371/journal.pone.0194970

Laist, D.W., 1987. Overview of the biological effects of lost and discarded plastic debris in the marine environment. Mar. Pollut. Bull. 18, 319-326. doi:http://dx.doi.org/10.1016/S0025-326X(87)80019-X 
Chapter 14. Analysis of microplastics in food samples

Li, J., Yang, D., Li, L., Jabeen, K., Shi, H., 2015. Microplastics in commercial bivalves from China. Environ. Pollut. 207, 190-195. doi:10.1016/j.envpol.2015.09.018

Liebezeit, G., Liebezeit, E., 2014. Synthetic particles as contaminants in German beers. Food Addit. Contam. - Part A Chem. Anal. Control. Expo. Risk Assess. 31, 1574 1578. doi:10.1080/19440049.2014.945099

Liebezeit, G., Liebezeit, E., 2013. Non-pollen particulates in honey and sugar. Food Addit. Contam. - Part A Chem. Anal. Control. Expo. Risk Assess. 30, 2136-2140. doi:10.1080/19440049.2013.843025

Lönnstedt, O.M., Eklöv, P., 2016. Environmentally relevant concentrations of microplastic particles influence larval fish ecology. Science (80-. ). 352, 1213 1216. doi:10.1126/science.aad 8828

Lu, Y., Zhang, Y., Deng, Y., Jiang, W., Zhao, Y., Geng, J., Ding, L., Ren, H., 2016. Uptake and Accumulation of Polystyrene Microplastics in Zebrafish ( Danio rerio ) and Toxic Effects in Liver. Environ. Sci. Technol. 50, 4054-4060. doi:10.1021/acs.est.6b00183

Mathalon, A., Hill, P., 2014. Microplastic fibers in the intertidal ecosystem surrounding Halifax Harbor, Nova Scotia. Mar. Pollut. Bull. 81, 69-79. doi:10.1016/j.marpolbul.2014.02.018

Mato, Y., Isobe, T., Takada, H., Kanehiro, H., Ohtake, C., Kaminuma, T., 2001. Plastic Resin Pellets as a Transport Medium for Toxic Chemicals in the Marine Environment. Environ. Sci. Technol. 35, 318-324. doi:10.1021/es0010498

Mühlschlegel, P., Hauk, A., Walter, U., Sieber, R., 2017. Lack of evidence for 
Chapter 14. Analysis of microplastics in food samples

microplastic contamination in honey. Food Addit. Contam. - Part A Chem. Anal. Control. Expo. Risk Assess. 34, 1982-1989. doi:10.1080/19440049.2017.1347281

Oberbeckmann, S., Löder, M.G.J., Labrenz, M., 2015. Marine microplastic-associated biofilms - a review. Environ. Chem. 12, 551. doi:10.1071/EN15069

Peng, L., Fu, D., Qi, H., Lan, C.Q., Yu, H., Ge, C., 2020. Micro- and nano-plastics in marine environment: Source, distribution and threats - A review. Sci. Total Environ. doi:10.1016/j.scitotenv.2019.134254

PlasticsEurope, EPRO, 2016. Plastics - the Facts 2016 An analysis of european plastics production, demand and waste data, Plastics - the Facts 2016.

Rainieri, S., Barranco, A., 2019. Microplastics, a food safety issue? Trends Food Sci. Technol. 84, 55-57. doi:10.1016/j.tifs.2018.12.009

Renzi, M., Blašković, A., 2018. Litter \&amp; microplastics features in table salts from marine origin: Italian versus Croatian brands. Mar. Pollut. Bull. 135, 62-68. doi:10.1016/j.marpolbul.2018.06.065

Rios, L.M., Moore, C., Jones, P.R., 2007. Persistent organic pollutants carried by synthetic polymers in the ocean environment. Mar. Pollut. Bull. 54, 1230-1237. doi:10.1016/J.MARPOLBUL.2007.03.022

Rochman, C.M., Hoh, E., Kurobe, T., Teh, S.J., 2013. Ingested plastic transfers hazardous chemicals to fish and induces hepatic stress. Sci. Rep. 3, 3263. doi:10.1038/srep03263

Rubio, L., Marcos, R., Hernández, A., 2019. Potential adverse health effects of ingested micro- and nanoplastics on humans. Lessons learned from in vivo and in vitro 
Chapter 14. Analysis of microplastics in food samples

mammalian models. J. Toxicol. Environ. Heal. - Part B Crit. Rev. doi:10.1080/10937404.2019.1700598

Sarria-Villa, R.A., Gallo-Corredor, J.A., 2016. La gran problematica ambiental de los residuos plasticos : Microplasticos. J. Cienc. e Ing. 8, 21-27.

Senathirajah, K., Palanisami, T., 2019. How Much Microplastics Are We Ingesting?: Estimation of the Mass of Microplastics Ingested. / Plastic ingestion by people could be equating to a credit card a week / Featured news / Newsroom / The University of Newcastle, Australia [WWW Document]. URL https://www.newcastle.edu.au/newsroom/featured/plastic-ingestion-by-peoplecould-be-equating-to-a-credit-card-a-week/how-much-microplastics-are-weingesting-estimation-of-the-mass-of-microplastics-ingested (accessed 2.10.20).

Shan, J., Zhao, J., Zhang, Y., Liu, L., Wu, F., Wang, X., 2019. Simple and rapid detection of microplastics in seawater using hyperspectral imaging technology. Anal. Chim. Acta 1050, 161-168. doi:10.1016/j.aca.2018.11.008

Talvitie, J., Heinonen, M., Pääkkönen, J.P., Vahtera, E., Mikola, A., Setälä, O., Vahala, R., 2015. Do wastewater treatment plants act as a potential point source of microplastics? Preliminary study in the coastal Gulf of Finland, Baltic Sea. Water Sci. Technol. 72, 1495-1504. doi:10.2166/wst.2015.360

Tanaka, K., Takada, H., Yamashita, R., Mizukawa, K., Fukuwaka, M. aki, Watanuki, Y., 2013. Accumulation of plastic-derived chemicals in tissues of seabirds ingesting marine plastics. Mar. Pollut. Bull. 69, 219-222. doi:10.1016/j.marpolbul.2012.12.010

Toussaint, B., Raffael, B., Angers-Loustau, A., Gilliland, D., Kestens, V., Petrillo, M., 
Chapter 14. Analysis of microplastics in food samples

Rio-Echevarria, I.M., Van den Eede, G., 2019. Review of micro- and nanoplastic contamination in the food chain. Food Addit. Contam. Part A 36, 639-673. doi:10.1080/19440049.2019.1583381

Yang, D., Shi, H., Li, L., Li, J., Jabeen, K., Kolandhasamy, P., 2015. Microplastic Pollution in Table Salts from China. Environ. Sci. Technol. doi:10.1021/acs.est.5b03163 